\title{
A Cost-Efficient Radiation Monitoring System for Nuclear Sites: Designing and Implementation
}

\author{
Arfat Ahmad Khan ${ }^{1, *}$ and Faizan Ahmed Khan ${ }^{2}$ \\ ${ }^{1}$ Suranaree University of Technology, Nakhon Ratchasima, 30000, Thailand \\ ${ }^{2}$ University of Engineering and Technology, Lahore, 54000, Pakistan \\ *Corresponding Author: Arfat Ahmad Khan. Email: arfat@g.sut.ac.th \\ Received: 24 August 2021; Accepted: 26 September 2021
}

\begin{abstract}
Radiation monitoring is essential for examining and refraining the unwanted situations in the vicinity of nuclear plants as high levels of radiation are quite dangerous for human beings. Meanwhile, Wireless Sensor Networks (WSNs) are proved to be an auspicious candidate to address that issue. Actually, WSNs are pretty beneficial to monitor an area with the aim of avoiding undesirable situations. In this paper, we have designed and implemented a cost-efficient radiation and the temperature monitoring system. We have used ZigBee to develop the sensor nodes, and the sensor nodes are instilled with the radiation and the temperature sensors. In addition, the Geiger Muller Tube (GM Tube), which is usually a reliable detector to monitor nuclear sites, is used to monitor the radiations. The designed system consists of two sensor nodes and the main node. The experimental results validate the designed system, where the communication between the nodes and the main node along with the inter-nodal communication are examined in order to ensure the smooth-running operation of the designed system.
\end{abstract}

Keywords: Wireless sensor network; ZigBee; radiation monitoring; communication

\section{Introduction}

During the normal running operations of nuclear plants, it has been seen that these plants release some sort of radiations due to radioactive waste along with some other reasons. As a result, this phenomenon imposes a serious threat to the society and the people living near to nuclear plants. Therefore, it is essential to monitor the radiation level with the aim of ensuring the safety of workers working in such plants along with the people living near to such plants.

There are overall three main types of radiations, i.e., alpha, beta, and gamma radiations. In alpha radiation, there are total two neutrons and two protons being bound together in such a way that they create a helium-4 like structure. In terms of detection, a GM tube having a thin window at one end is used. In beta radiation, during the decaying of beta, a positron or electron are released due to the decaying of an atomic nucleus. Moreover, the pancake shaped GM probes are used for the detection/ monitoring of beta radiations. Gamma radiations, also called as electromagnetic radiations, are emitted

This work is licensed under a Creative Commons Attribution 4.0 International License, which permits unrestricted use, distribution, and reproduction in any medium, provided the original work is properly cited. 
from an excited nucleus, and these radiations have the shortest wavelength along with the highest frequency. Gamma radiations can be detected with the help of Geiger counters.

In terms of absorbing the dose, it is dependent on the material. Moreover, the effects of radiation on the material depends on the environment conditions as the radiations of large magnitude cause more damage as compared to the radiations of less magnitude. Furthermore, the Geiger Muller Tube (GM Tube), which is usually a reliable detector to monitor the nuclear sites, is used to monitor the radiations. The designed system comprises of a main node along with the two sensor nodes. The output of the GM Tube is analyzed with the help of an oscilloscope. The final results unveil the smooth-running operations of the designed system where the communication between the nodes and the main node along with the internodal communication (from the node A to the node B \& from the node B to the node A) is examined in order to ensure the smooth-running operation of the designed system.

\section{Literature Review}

In Wireless Sensor Networks (WSNs), there are large number of geographically distributed sensor nodes to monitor environment parameters (indoor as well as outdoor) [1,2]. These sensor nodes are usually low cost and are capable to sense the environment along with sending the sensed data to the respective base station [3]. In fact, we can gather the required data from the sensor nodes by inserting our required queries because these nodes are instilled with their own communication devices [4,5]. These nodes are also capable for organizing themselves on their own following their deployment. To retrieve the location of these nodes, we use Global Positioning System (GPS) [6].

In terms of communication, there are various standards used in WSNs such as ZigBee, Bluetooth, Long range wide area network (LoRa WAN) and Sigfox. ZigBee requires low power consumptions and cost and are capable to deliver services such as e-mail, Internet, and the transfer of files [7], which brings a lot of benefits by considering the fact that the internet data traffic is increasing with every passing day $[8,9]$. Bluetooth also requires low power consumptions and is a cost-efficient device; however, it has less range compared to the ZigBee modules [10]. LoRa, a new ISM band technology, is used for long range operations [11].

As far as applications are concerned, WSNs are used for a wide range of applications, i.e., industrial applications, environment monitoring applications, military applications, and medical applications. In [12], the authors have developed a real time monitoring application in a coffee factory based on WSNs by considering both the perspectives i.e., hardware as well as software. In [13], the authors have proposed an air monitoring system based on WSNs for smart industries. The concentrations of sulfur dioxide (SO2), carbon monoxide (CO), and dust are monitored in their air monitoring system. In [14], authors have used WSNs for monitoring data in a forest. Actually, the real time parameters are collected, analyzed and graphically visualized with the aim of monitoring the condition of the forest. In [15], the authors have designed an agricultural monitoring system with the aim of monitoring the environment parameters. Actually, the authors deploy 24 nodes based on ZigBee technology by using the software OPNET. In [16], the authors have developed a water quality monitoring system based on WSNs. The values of turbidity, $\mathrm{pH}$ and the density of oxygen are sent from the sensor networks to the base station.

In [17], the authors develop a real time monitoring system for the monitoring of nuclear power plants. The applications of WSNs are highlighted especially for tackling the undesirable situations in nuclear power plants. In [18], the authors have proposed a web based remote monitoring system with the aim of ensuring the safety of workers worked in nuclear plants. The ZigBee modules powered by UPS are used to transmit the data. In [19], the authors have presented a radiation monitoring system for nuclear plants. The ZigBee device is used to monitor the radiation because of its myriad benefits, i.e., low cost, scalability, low energy and power consumptions and the transmission of data in a reliable way. However, the authors only use a 
single node for the monitoring of radiations and the research is devoid of inter-nodal communications. In [20] the authors deploy the centralized system with the aim of monitoring the radiations. The research is mainly aimed to compute the optimal number of router nodes by considering the performance of the network.

In this paper, we have worked on designing and the implementation of a cost-efficient radiation and the temperature monitoring system. During the development of sensor nodes, we opted for ZigBee as it requires low power consumptions and cost and are capable to deliver services such as e-mail, Internet, and the transfer of files. Moreover, the sensor nodes are instilled with the temperature as well as the temperature sensors. Furthermore, the Geiger Muller Tube (GM Tube), which is usually a reliable detector to monitor the nuclear sites, is used to monitor the radiations. The designed system comprises of a main node along with the two sensor nodes. The output of the GM Tube is analyzed with the help of an oscilloscope. The final results unveil the smooth-running operations of the designed system where the communication between the nodes and the main node along with the inter-nodal communication (from the node A to the node B $\&$ from the node B to the node A) is examined in order to ensure the smooth-running operation of the designed system.

The rest of the paper is organized as follows: The Section 3 contains designing and the hardware configuration, and the simulations and experimental results are discussed in the Section 4 and 5, respectively. Finally, the Section 6 concludes the research work.

\section{Designing and the Hardware Configuration}

During the designing of the hardware, we have opted for the cost efficient hardware components, communication modules along with the nodes. In addition, by comparing the price of commercially available expensive radiation monitoring system, the cost of the designed system is significantly less. The main components of the hardware are shown in the Tab. 1. To display the temperature and counts per minute, we have used the LM016L.

Table 1: Main components

\begin{tabular}{ll}
\hline Component & Value \\
\hline LCD & LM016L \\
Microcontroller & $18 \mathrm{f} 452$ \\
ZigBee & S1 module \\
Temp Sensor & LM35 \\
GM Tube & ZP 1400 \\
Nodes & 2 \\
Main Node & 1 \\
\hline
\end{tabular}

Actually, the LM016L has 2 rows and 16 columns. The LCD was not mandatory; however, we used it with the aim of monitoring the processing of microprocessors. The Fig. 1 unveils the pin configuration.

In addition, in order to control the processing, we have used PIC microcontroller because of its available nonvolatile memory. We connected the LCD to the port B and pins (TX and RX) are used to connect the ZigBee module. The Fig. 2 and Tab. 2 unveil the pin configuration and specifications, respectively. 

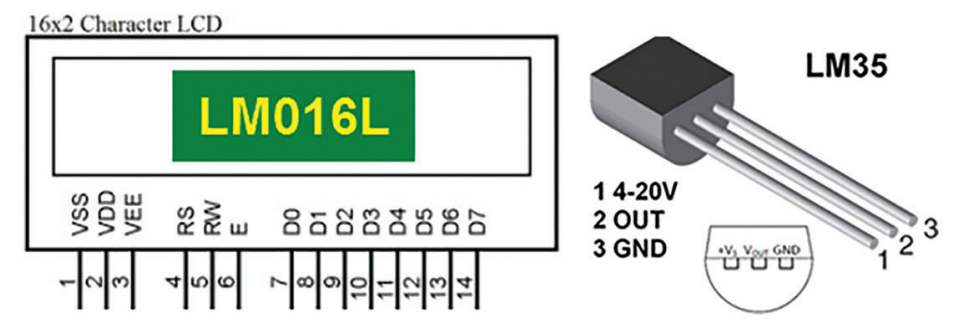

Figure 1: Pin configuration of LCD and the temperature sensor

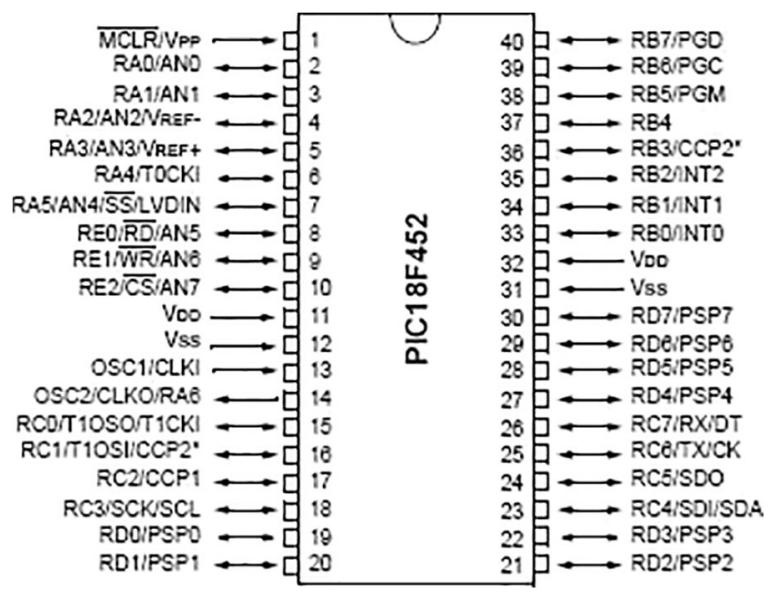

Figure 2: Microcontroller pin configuration

Table 2: Microcontroller specifications

\begin{tabular}{ll}
\hline Component & Value \\
\hline Program Memory Type & Flash \\
Program Memory (KB) & 32 \\
CPU Speed (MIPS) & 10 \\
RAM Bytes & 1536 \\
Data EEPROM (bytes) & 256 \\
PWM & 2 \\
Timers & 4 \\
ADC & 10 Bit \\
Temperature Range (C) & $-40-125$ \\
Voltage (operating) & $2-5 \mathrm{~V}$ \\
Pin Count & $40 \mathrm{~V}$ \\
\hline
\end{tabular}

For the personal area network, we used S1 modules of ZigBee which has the power and baud rate of $1 \mathrm{~mW}$ and 9600, respectively. The Fig. 3 unveils the data flow between the microcontroller and the ZigBee modules. 


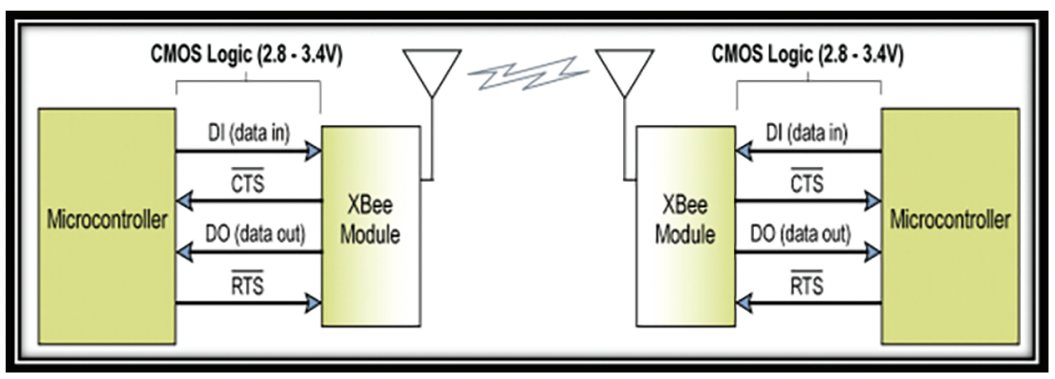

Figure 3: Data flow between the microcontroller and the ZigBee

To monitor the temperature and radiation, we used LM 35 (shown in the Fig. 1) and GM tube ZP1400 (internal configuration is shown in the Fig. 4) to monitor the temperature and radiation, respectively.

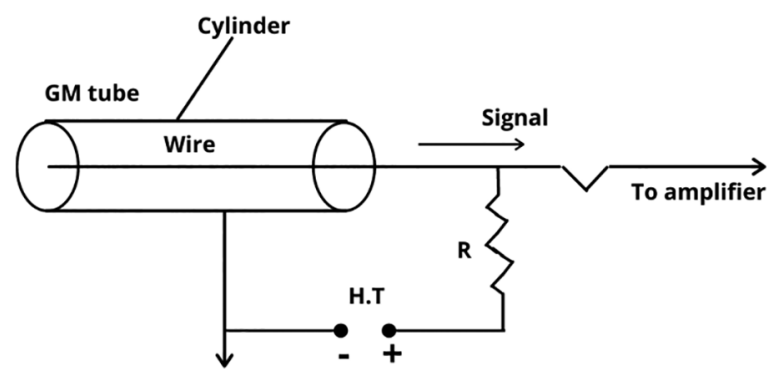

Figure 4: GM tube

The Fig. 5 unveils the final hardware, where it can be seen that there are total two nodes (for monitoring the temperature and radiations) along with the main node. Actually, the GM tube is utilized for the detection and monitoring of almost all types of radiations as it uses a pair of electrodes. Moreover, Argon or Helium gas is used to surround the electrodes.

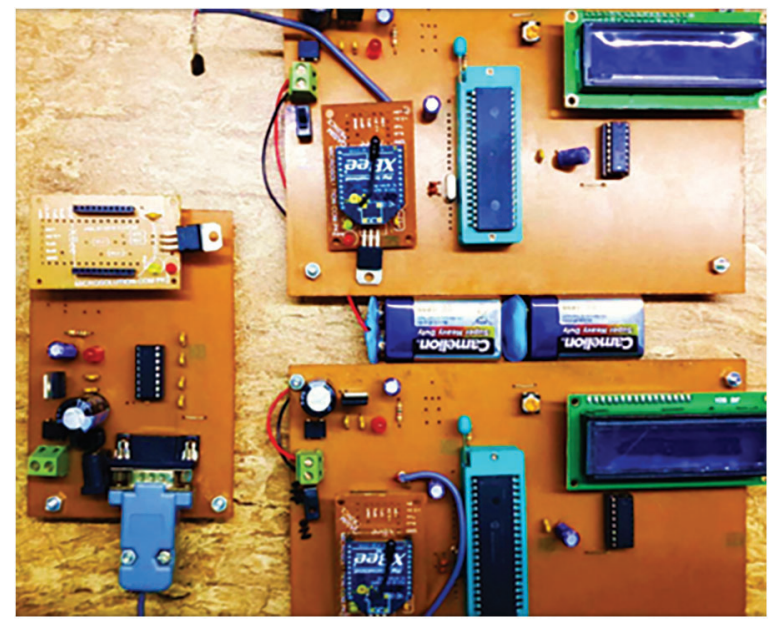

Figure 5: Final hardware 


\section{Simulations}

To test the working of the project, we used Proteus, which provides the real time simulations, by connecting all the components required for this project. Moreover, it also provides vitual terminals to connect the ZigBee modules. The main reason of choosing this software is its ability to connect the software designing with the hardware designing. The Fig. 6 shows the connections on the software.

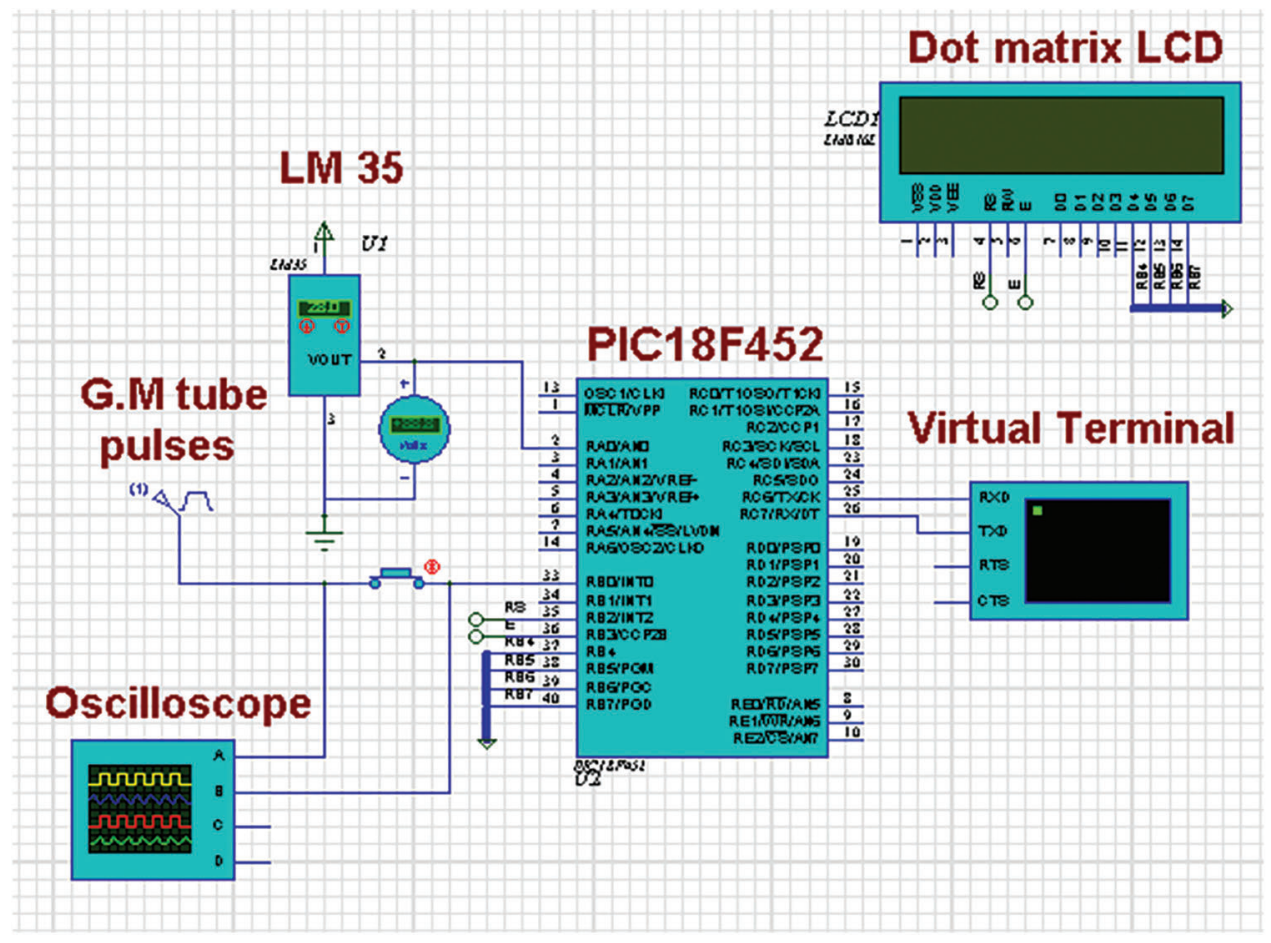

Figure 6: Implementation on proteus

In addition, we used mikro $\mathrm{C}$ for the coding because it creates HEX file which can be given to PIC controllers by using Proteus. The main flow of the coding is unveiled in the Fig. 7.

Actually, Coordinator software flow diagram depicts the condition of joining a new node in Personal area network (PAN). Every node is assigned a new 16 bit address to it. Coordinator continuously searches whether a new node is present in that area or not. Moreover, Node continuously measures the input temperature and count pulses through interrupt port. It repeats this process and finally sends the data using ZigBee communication. To count through interrupt port (port B1.0) we must take care of clear flag and interrupt flag. Interrupt detect any pulse if it is above 2.2 volts. Whenever pulse arrives, it increment one to the counter. By setting in prescaler bits we can count max of 500 counts per second which is more than maximum limit of our radiation environment.

\section{Results and Discussion}

In this section, we have presented and discussed the experiment results. The Fig. 8 unveils the output voltage of 4 pin DMM IC on the multimeter, where it can be seen that the output of the IC comes out be 500 volts by applying 2.2 volts at the input. 


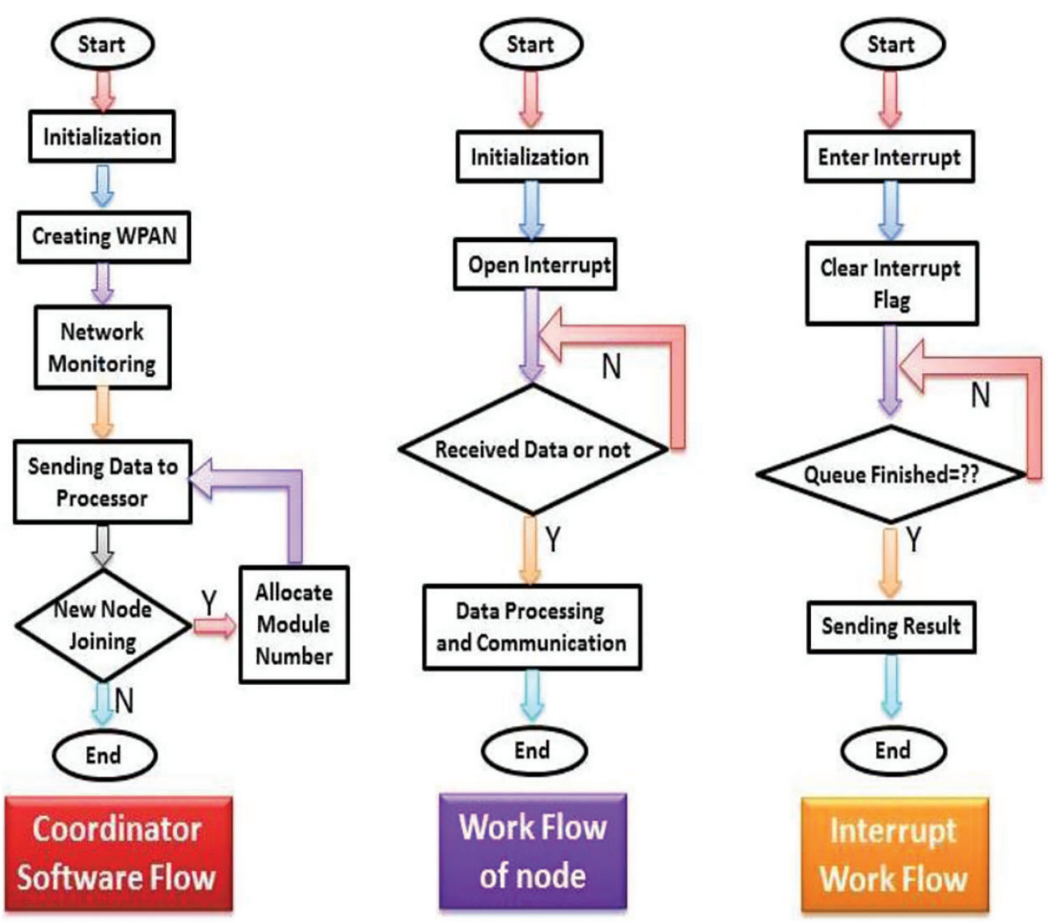

Figure 7: Work flow

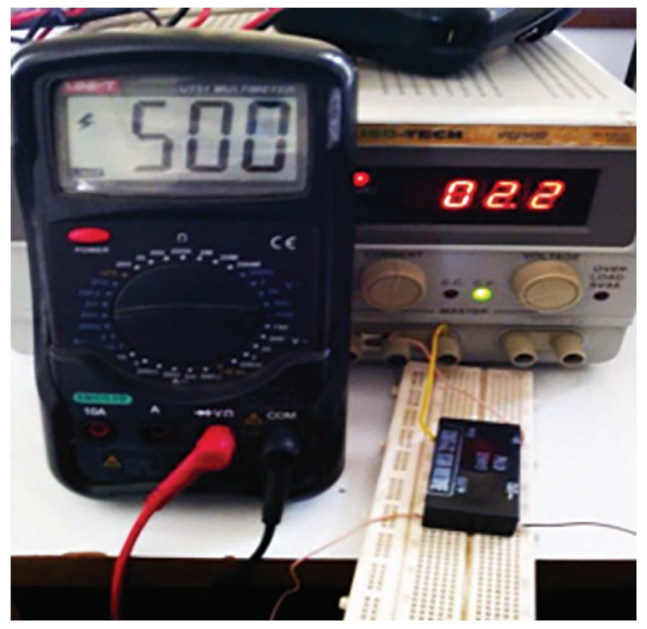

Figure 8: Testing DMM IC

The Fig. 9 shows the connection of DMM IC with the GM tube in order to test the working. The output of the GM is further evaluated by using the oscilloscope as can be seen in the Fig. 10. The voltage gets reduced by utilizing the capacitor because the sensor got the built-in capacitance. The output voltage comes out to be $2.2 \mathrm{mv}$ when the sensor is devoid of capacitance.

The connectivity between the nodes along with the communication between the nodes with main node is also examined to ensure the smooth-running operation of the system. To test the connectivity between the node A and the main node, the letter A was sent by using the Audrino software. Following the few seconds, we received the node A's data as can be seen in the Fig. 11. 


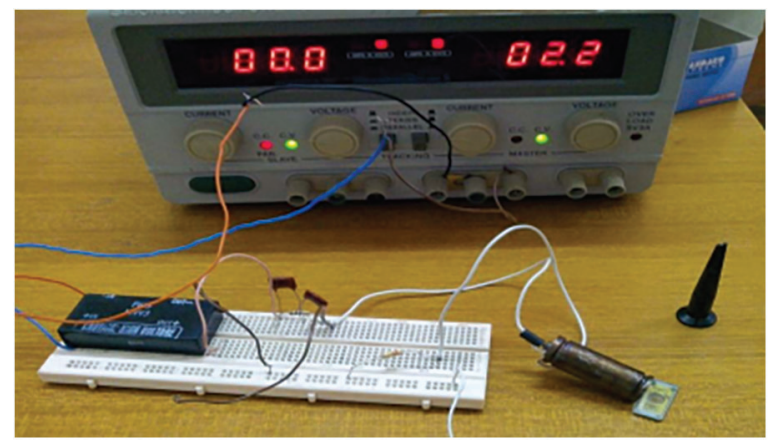

Figure 9: Testing the radiation sensor
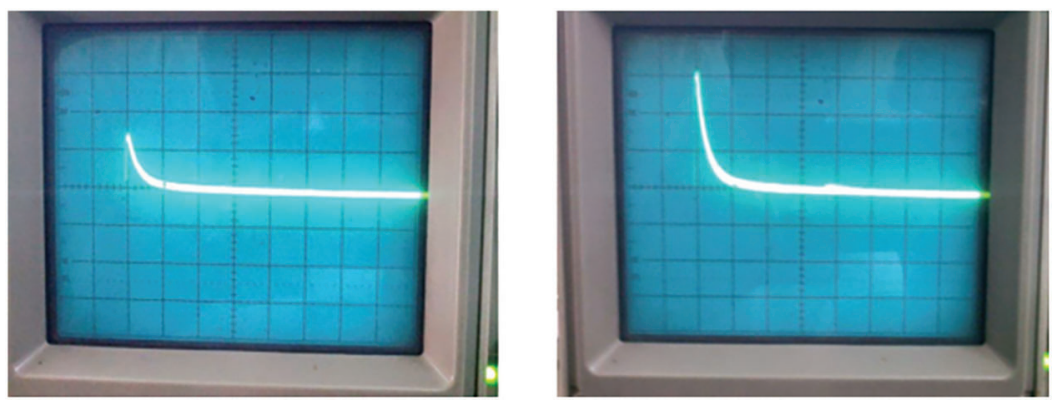

Figure 10: Examining the response of the radiation sensor by using oscilloscope
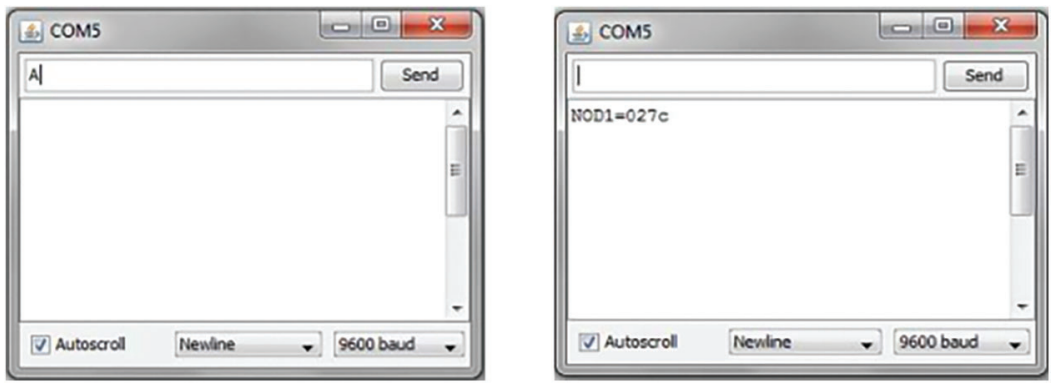

Figure 11: Communication between the node $A$ and the main node

Similarly, to ensure the connectivity between the node B and the main node, the letter E was sent by using the Audrino software. Following the few seconds, we received the node B's data as can be unveiled in the Fig. 12.
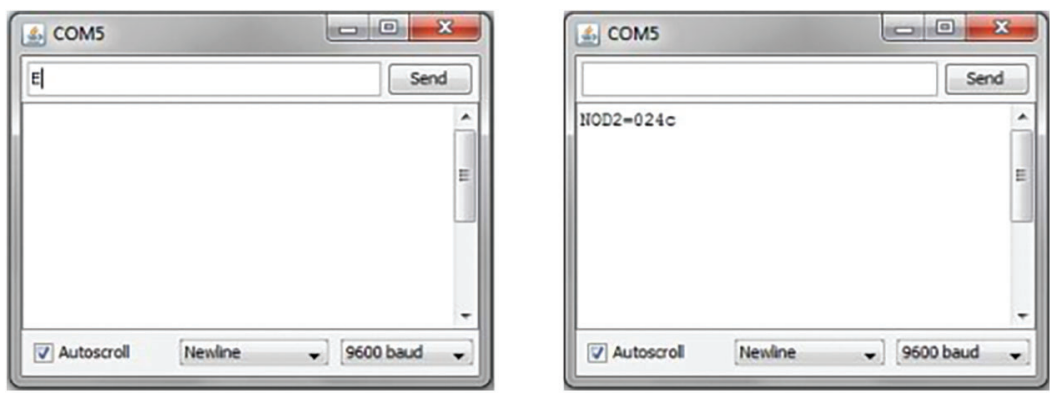

Figure 12: Communication between the node $B$ and the main node 
The Fig. 13 shows the communication from the Node A to Node B by sending letter B in the windows. It is vital to mention that the $\mathrm{CD}$ flag was used from the node $\mathrm{A}$ to node $\mathrm{B}$ communication.
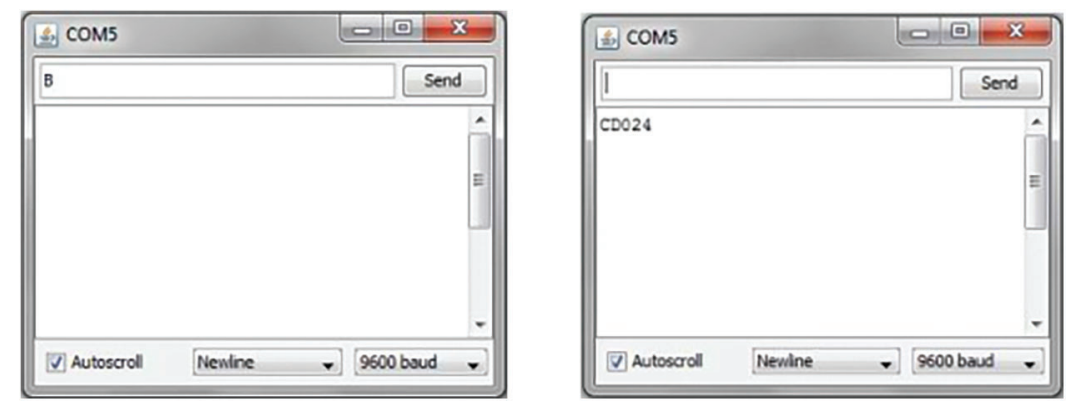

Figure 13: Communication from the node A to the node B

Likewise, the Fig. 14 shows the communication from the Node B to Node A by sending letter F in the windows. It is vital to mention that the GH flag was used from the node B to node A communication. The Tab. 3 summarizes the addressing and the used flags for communications.
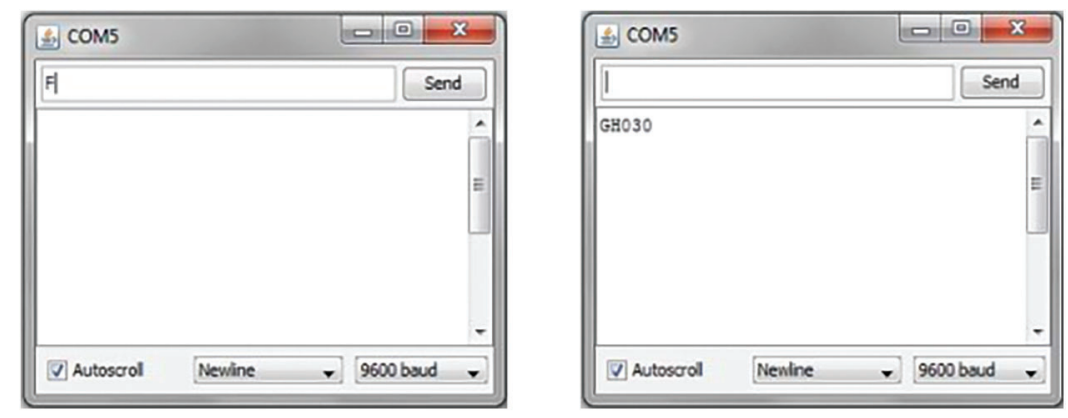

Figure 14: Communication from the node $B$ to the node A

Table 3: Addressing map

\begin{tabular}{ll}
\hline Addressing & Response \\
\hline A & Communication between the Node A and the Main Node. \\
B & Communication from the Node A to Node B by using the flag CD \\
E & Communication between the Node B and the Main Node. \\
F & Communication from the Node B to Node A by using the flag GH \\
\hline
\end{tabular}

\section{Conclusions}

The paper mainly worked on designing and the implementation of a cost-efficient radiation as well as the temperature monitoring system. By considering the advantages of ZigBee modules, we used them to send the data of the radiation sensor as well as the temperature sensor. The ZigBee modules are extremely useful for the monitoring applications as the physical monitoring of the nuclear sites can significantly damage the life of humans. In addition, the output of the GM tube was analyzed with the help of oscilloscope, where we noticed that the voltage got reduced by utilizing the capacitor because the sensor got the built-in capacitance. The output voltage came out to be $2.2 \mathrm{mv}$ when the sensor was devoid of capacitance. The results unveiled 
the smooth-running operations of the designed system where the communication between the nodes and the main node along with the inter nodal communication (from the node $\mathrm{A}$ to the node $\mathrm{B} \&$ from the node $\mathrm{B}$ to the node A) were examined in order to ensure the smooth-running operation of the designed system. The CD flag was used from the node A to node B communication, and the GH flag was used from the node B to node A communication. In terms of future recommendations, the developed system can be further improved by adding more sensors, i.e. pressure and the humidity sensor. Moreover, we can also equipped the system with the security modules in order to protect the communication between the nodes and the main system.

Funding Statement: This research was supported by Suranaree University of Technology, Thailand.

Conflicts of Interest: The authors declare that they have no conflicts of interest to report regarding the present study.

\section{References}

[1] J. Chen, Y. C. Wu and W. Ouyang, "Efficient wireless charging pad deployment in wireless rechargeable sensor networks," IEEE Access, vol. 8, pp. 39056-39077, 2020.

[2] X. Jiazu, B. Zhang and C. Zhang, "A novel relay node placement and energy efficient routing method for heterogeneous wireless sensor networks," IEEE Access, vol. 8, pp. 202439-202444, 2020.

[3] J. Mao, X. Jiang and X. Zhang, "Analysis of node deployment in wireless sensor networks in warehouse environment monitoring systems," EURASIP Journal on Wireless Communications and Networking, vol. 2019, no. 1, pp. 10, 2019.

[4] A. A. Khan, P. Uthansakul, P. Duangmanee and M. Uthansakul, "Energy efficient design of Massive MIMO by considering the effects of nonlinear amplifiers," Energies, vol. 11, no. 5, pp. 1045, 2018.

[5] P. Uthansakul and A. A. Khan, "Enhancing the energy efficiency of mmWave Massive MIMO by modifying the RF circuit configuration," Energies, vol. 12, no. 22, pp. 4356, 2019.

[6] B. Buchli, F. Sutton and J. Beutel, "GPS-equipped Wireless sensor network node for high-accuracy positioning applications," in Proc. of European Conf. on Wireless Sensor Networks, Trento, Italy, 2012.

[7] J. Lee and Y. Wang, "Experimental evaluation of ZigBee-based wireless networks in indoor environments," Journal of Engineering, vol. 2013, no. 4, pp. 1-9, 2013.

[8] P. Uthansakul and A. A. Khan, "On the energy efficiency of millimeter wave Massive MIMO based on hybrid architecture," Energies, vol. 12, no. 11, pp. 2227, 2019.

[9] A. A. Khan, P. Uthansakul and M. Uthansakul, "Energy efficient design of Massive MIMO by incorporating with mutual coupling," International Journal on Communications Antenna and Propagation (IRECAP), vol. 7, no. 3, pp. 198-207, 2017.

[10] D. Hortelano, T. Olivares, M. C. Ruiz, C. Garrido-Hidalgo and V. López, "From sensor networks to Internet of Things, bluetooth low energy, a Standard for this evolution," Sensors, vol. 17, no. 2, pp. 372, 2017.

[11] A. J. Wixted, P. Kinnaird, H. Larijani, A. Tait, A. Ahmadinia et al., "Evaluation of LoRa and LoRa WAN for wireless sensor networks," in Proc. of 2016 IEEE Sensors, FL, USA, pp. 1-3, 2012.

[12] J. Valverde, V. Rosello, G. Mujica, J. Portilla, A. Uriarte et al., "Wireless sensor network for environmental monitoring: Application in a coffee factory," International Journal of Distributed Sensor Networks, vol. 8, no. 1, pp. 638067, 2012.

[13] G. Swagarya, S. Kaijage and R. S. Sinde, "Air pollution monitoring system based on wireless networkssimulation," Innovative Systems Design and Engineering, vol. 5, pp. 9-15, 2014.

[14] A. Awang and M. H. Suhaimi, "RIMBAMONO: A forest monitoring system using wireless sensor networks," in Proc. of 2007 Int. Conf. on Intelligent and Advanced Systems, Kuala Lumpur, Malaysia, pp. 1101-1106, 2017.

[15] M. Keshtgari, "A wireless sensor network solution for precision agriculture based on zigbee technology," Wireless Sensor Network, vol. 4, no. 1, pp. 25-30, 2012. 
[16] R. Yue and T. Ying, "A water quality monitoring system based on wireless sensor network \& solar power supply," in Proc. of the 2011 IEEE Int. Conf. on Cyber Technology in Automation, Control, and Intelligent Systems, Kunming, China, pp. 126-129, 2011.

[17] R. Lin, Z. Wang and Y. Sun, "Wireless sensor networks solutions for real time monitoring of nuclear power plant," in Proc. of the 5 World Congress on intelligent Control and Automation, Hangzhou, China, pp. 3663-3667, 2004.

[18] L. Wang, L. Wu, Q. Liu, Li. Shen, Y. Dong et al., "Web based remote monitoring system for nuclear power plant," in Proc. of the 2014 4th IEEE Int. Conf. on Information Science and Technology, Shenzhen, China, pp. 196-199, 2014.

[19] L. Wu, L. Wang, Q. Liu, L. Shen, S. Su et al., "Wireless sensor network based solution for nuclear radiation detection," in Proc. of the 2014 4th IEEE Int. Conf. on Information Science and Technology, Shenzhen, China, pp. 2164-4357, 2014.

[20] J. Ebenezer and S. S. Murty, "Deployment of wireless sensor Network for radiation monitoring," in Int. Conf. on Computing and Network Communications (CoCoNet), Trivandrum, India, pp. 27-32, 2015. 